\title{
Rhodotoruliosis: An Emerginag Opportunistic Mycosis of Humans and Animals
}

\author{
Mahendra Pal ${ }^{1}$, Rodrigues Paula $C^{2}$, da Silva Ruiz L ${ }^{3}$ and Paulos Gutama $\mathrm{K}^{4}$ \\ ${ }^{1}$ Narayan Consultancy on Veterinary Public Health and Microbiology, India \\ ${ }^{2}$ School of Dentistry, University of Sao Paulo, Brazil \\ ${ }^{3}$ Adolfo Lutz Institute, Brazil \\ ${ }^{4}$ Adaba District Livestock and Fishery Development and Resource Office, Ethiopia
}

Mini Review

Volume 4 Issue 2

Received Date: October 21, 2021

Published Date: November 15, 2021

DOI: $10.23880 /$ oajmms-16000148

*Corresponding author: Mahendra Pal, Founder Director of Narayan Consultancy on Veterinary Public Health and Microbiology, Gujarat, India, Email: palmahendra2@gmail.com

\section{Abstract}

Rhodotoruliosis is an opportunistic emerging mycotic disease that affects both humans and animals. The source of infection is exogenous; and disease can occur in sporadic as well as epidemic form. The disease is caused by Rhodotorula, basidiomycetous yeast that lives as a saprophyte in the environment. Rhodotorula glutinis, $R$. mucilaginosa (R.rubra), and R. minuta are the most frequently encountered species of Rhodotorula. The species can be isolated from a wide range of sources in nature, including the soil, air, ocean, plants, dairy products, and the household environment. Fungemia, meningitis, ventriculitis, peritonitis, endocarditis, keratitis, endophtalmitis, hydrosalpinx, oral ulcer and lymphadenitis have all been linked to the fungus in humans. It causes skin infections in chicken, sea lion, and cat, lung infection in sheep, epididymitis in dog, and mastitis in cow and buffalo. The organism is an opportunist, taking advantage of immunosuppressive circumstances, indwelling devices, and antibiotic exposure. In order to confirm the diagnosis of rhodotoruliosis, laboratory assistance is required. The direct demonstration of Rhodotorula in the clinical specimens and its isolation in pure and luxuriant growth still considered the gold standard of diagnosis. Amphotericin B or one of its lipid formulations appears to be the medication of choice for treating the patient. The infection due to Rhodotorula especially in immunosuppressed individuals can be life threatening if the treatment is delayed. Prognosis of systemic disease in compromised subject may be grave. Early diagnosis is essential to start specific antifungal therapy of rhodotorulosiosis to prevent serious complications.

Keywords: Animals, Basidiomycetous yeast, Emerging pathogen, Humans, Immunocompromised host, Rhodotorula

\section{Introduction}

In the last few decades, fungus infections linked to healthcare assistance have become a major medical concern. Although Candida and Aspergillus species cause the bulk of these infections, a growing proportion of infections are caused by less prevalent pathogens [1]. Some authors have referred to these fungi as "emerging pathogens." Historically, many of them were thought to be laboratory contaminants and/or of poor virulence. Yeasts other than Candida species, as well as a wide range of dematiaceous and hyaline moulds, are among these pathogens [2].

Rhodotoruliosis is a sporadic, non-contagious, opportunistic mycotic disease of humans and animals [3]. Rhodotorula, basidiomycetous yeast, is found in nature; and has been isolated from several environmental sources as well as dairy products [3-5]. It was once thought to be non-pathogenic. It has emerged as an opportunistic etiological agent in the last two decades, particularly in immunocompromised hosts. They can also be present in the skin, nails, and mucous membranes as commensal yeasts [2]. 


\section{Open Access Journal of Mycology \& Mycological Sciences}

It is important to mention that meningitis, fungemia, ventriculitis, endocarditis, peritonitis, endocarditis, endophtalmitis, keratitis, lymphadeniis, oral ulcer and infections of devices, such as catheters and contact lenses have all been related to Rhodotorula [3,6-8]. It is also been found as a saprophyte on the skin, vaginal, and respiratory specimens, as well as a colonizing organism on hemodialysis machines and bronchoscopes [9,10]. Rhodotorula species have been linked to clinical mastitis in dairy cows and buffaloes in animals $[3,5,11,12]$, a reproductive problem [13] and gastrointestinal infection in calves [11]. The prime objective of this mini review is to promote awareness of rhodotoruliosis as an emerging mycotic disease of humans as well as animals.

\section{Etiology}

Rhodotorula is basidiomycetous yeast belonging to the phylum Basidiomycota and the family Sporidiobolaceae [14]. The genus Rhodotorula has 34 species of which Rhodotorula glutinis, $R$. mucilaginosa, and $R$. minuta are the most frequent Rhodotorula species [15]. Rhodotorula species are nutritionally non-fastidious, grow well on a variety of media, and have a quick growth rate. The morphology of the colony has been described as soft, smooth, moist, and occasionally mucoid. Pseudohyphae are rare, and they appear as round or oval budding cells under microscopy. Occasionally, a faint capsule forms. Urease is produced by Rhodotorula species, although they do not ferment carbohydrates. Due to the presence of carotenoid pigments, most Rhodotorula species generate pink to coral colonies on Sabouraud dextrose agar, although they can also be orange to red on Sabouraud dextrose agar [16].

\section{Host and Transmission}

Natural infection due to Rhototorula species has been described in humans and in many species of animals, such as buffalo, cat, chicken, cow, dog, goat, pig, sea lion, and sheep [3,5]. Recently, Dave P, Pal M [5] have isolated Rhototorula from the pigeon droppings on APRM medium. The source of infection is exogenous and the infection may be acquired through respiratory tract. However, the fungus can also enter the body of the host via wound, abrasion or injury on the skin [3].

\section{Pathogenesis}

The pathophysiology of Rhodotorula infection has not been investigated. As previously stated, there is nearly always underlying immunosuppression and/or the presence of a foreign body [17]. Invasive infections may occur as a result of environmental contamination of an inserted prosthetic device, but it appears more likely that the organism is an opportunist that colonizes and infects at-risk patients by taking advantage of immunecompromising conditions, indwelling devices, and exposure to broad-spectrum antibiotics $[18,19]$.

\section{Epidemiology}

Rhodotoruliosis is an emerging opportunistic mycosis [17], and is reported from several countries of the world including India [8,20-23]. The disease often occurs in sporadic form, however, outbreak has also been recorded $[3,21,23,24]$. Rhodotorula species are implicated as a cause fungaemia, endocarditis, meningitis, peritonitis, keratitis, oral ulcers, and central venous catheter infection $[7,8,25]$. Rhodototorula has been isolated from the conjunctival swab of a person by Dave P, et al. [5].

Rhodotorula infection has been reported in humans, and several species of animals, such as buffalo, cat, chicken, cow, dog, goat, pig, and sheep $[3,5]$. The species can be isolated from a range of sources in nature, including air, soil, saltwater, plants, dairy products, and the home environment (e.g., shower curtains, bathtub grout) [1,3]. It is also possible that this opportunistic fungus can contaminate laboratory specimens. Rhodotorula species have been found in human skin, nails, respiratory, gastrointestinal, and urinary tract cultures, and they are assumed to be commensals [26].

Rhototorula mucilaginosa is prominent basidiomycetous yeast that is found in a variety of natural habitats, including living or decomposing plant constituents, soil, and diverse aquatic environments, including fresh waters, estuaries, and coastal waters, as well as Open Ocean and deep sea environments. It can also be found in harsh conditions, such as hyper acidic waters and uranium environs [27]. Rhototorula mucilaginosa has been found in peanuts, apple cider, cherries, fresh fruits, fruit juices, cheeses, sausages, edible mollusks, and crustaceans. Although eating yeastcontaminated food may not cause opportunistic infection directly, there is growing worry that food may be an underappreciated source of environmental pathogens [17].

Rhodotorula glutinis complex has also been isolated from a range of substrates and is found all over the world. Air, fresh water, sea water, terrestrial settings, food and beverages, animals, and humans are all known sources of this saprobic fungus. Rhodotorula minuta has been recovered less frequently in natural environments than $R$. rubra and R. glutinis complex. This species was found in the air, in sea water (including the deep sea), and in fresh water [14]. Infection due to Rhodotorula can occur through inhalation and also by accidental inoculation of the fungus through abraded skin [3]. Recently, Perniola and co-workers [24] recorded an outbreak of Rhodotorula mucilaginosa in 


\section{Open Access Journal of Mycology \& Mycological Sciences}

neonatal intensive care unit.

\section{Clinical spectrum}

\section{Humans}

Because Rhodotorula is such a common and saprophytic fungus, its isolation from nonsterile human sites, particularly mucosal membranes, has been a source of debate in the medical community [17]. Rhodotorula species have been linked to a variety of diseases, including fungaemia [28]. The first report of fungemia caused by Rhodotorula was published in 1960 [29]. Increased use of more aggressive treatment modalities, such as intensive care unit admissions, central venous catheter use, short- and long-term parenteral nutrition, broad-spectrum antibiotics, organ transplants, and chemotherapy, was related to an increase in Rhodotorula fungemia linked to catheters [28]. Endocarditis, meningitis, ventriculitis, keratitis, endophtalmitis, and peritonitis are some of the other clinical symptoms others than fungaemia [8]. There is also a case of lymphadenitis attributed due to Rhodotorula mucilaginosa in a man with well-controlled HIV infection [23] and a case of pelvic infection with bilateral hydrosalpinx was caused by Rodotorula glutinis [30]. The association of Rhodotorula mucilaginosa in oral ulcer of a AIDS patient is reported by Kaur R, et al. [7]. It is mentioned that majority of infections due to Rhototorula were fungaemia [17].

\section{Animals}

Several instances of an outbreak of the skin infections in chickens are among the rare references about the pathogenicity of Rhodotorula spp. in animals [31] and a report of a lung infection in sheep was caused by $R$. mucilaginosa [32]. Rhodotorula has been linked to epididymitis in dogs [33], skin lesions in a sea lion [34], dermatitis in a cat with crusted lesions, and mastitis in cows and buffaloes [3,5,35]. Duarte ER, et al. [36] has shown the presence of fungi in the ear canal of 45 cattle with external parasitic otitis. Similarly, Rhodotorula genus can colonize the ear canals of adult calves suffering from parasitic otitis [37]. Organs, such as the lungs, spleen, and notably the liver were the most severely afflicted. Given the animals' immunocompromised state, histology of the affected organs revealed few epitelioid cells and multinuclear giant cells, as well as numerous yeast forms and the occasional formation of granulomas [17].

\section{Diagnosis}

Isolation of Rhodotorula species from non-sterile sites such as skin, sputum, or stool is more likely to be due to colonization or contamination, and therapy should be initiated only if symptoms strongly indicative of infection are present and all other reasons have been ruled out [26]. On Sabouraud dextrose agar with chloramphenicol, Pal M. [38] sunflower seed medium and APRM [Anubha, Pratibha, Raj, Mahendra] [5], the fungus can be easily isolated from clinical specimens. The microscopic morphology of Rhodotorula isolates is studied by using PHOL Pal M, et al. [39], or the Narayan stain, which includes $6 \mathrm{~mL}$ dimethylsuloxide, $4.0 \mathrm{~mL}$ glycerin, and $0.5 \mathrm{~mL} 3$ percent methylene blue solution [40]. As Pal sunflower seed medium, APRM agar, PHOL stain and Narayan stain are cheaper than other media and stains, it is therefore, advised that Microbiology and Public Health laboratories should routinely employ these media and stains for the study of fungi including Rhodotorula.

Rhodotorula species found in sterile environments, such as blood, peritoneal fluid, or CSF are frequently symptomatic of infection. Since yeast cells may usually be observed on microscopic examination, morphological and biochemical confirmation of the diagnosis should be sought. They differ from Cryptococcus species in that they cannot assimilate inositol, and they differ from Candida species in that they produce colored colonies and lack pseudohyphae [16]. Colony coloration, confirming biochemical tests (e.g., lack of carbohydrate fermentation, urease production), and the absence of ballistospore generation should all lead to a particular mycological identification of Rhodotorula [21,22].

\section{Treatment}

In animals, no treatment has been recommended [3]. The therapy of Rhodotorula fungemia in humans is still debatable. Recovery requires the resolution of coexisting neutropenia. The removal of the central venous catheter is usually sufficient, and systemic antifungal treatment is rarely necessary [41]. Rhodotorula is a low-virulence fungus with a low mortality rate. As a result, most Rhodotorula fungemia patients in the literature survived with or without antifungal treatment. Amphotericin B or one of its lipid formulations appears to be the medication of choice based on available in vitro susceptibility data [8]. The most effective antifungal agents against $R$. mucilaginosa are amphotericin $\mathrm{B}$ and flucytosine. Fluconazole resistance has been observed in all strains of Rhodotorula. Cross resistance to other azole medications is not common, however, itraconazole, voriconazole, and posaconazole resistance is found in more than $60 \%$ of the strains. This yeast species is also resistant to echinocandins $[8,42]$.

\section{Prevention and Control}

Minimizing related risk factors is an important part of preventing Rhodotorula species infections. Patients infected with Rhodotorula species do not need to take any special precautions to prevent the infection. The organisms 


\section{Open Access Journal of Mycology \& Mycological Sciences}

are found in abundance in the environment and are most likely acquired by colonization with environmental strains. There is no indication of Rhodotorula species transmission from person to person. Rhodotorula spp. can, however, infect health-care professionals' hands (as well as any rings). Standard infection control precautions, such as hand washing and thorough skin cleansing and preparation prior to invasive treatments, should be emphasized in the absence of particular evidence [43]. As the fungus can enter through the abraded skin, it is, therefore, advised to avoid traumatic injury to the skin. Further, proper medical attention should be given to skin injury [44]. In immunocompromised patients, early identification and chemotherapy are critical for preventing disease spread. Mastitis can be reduced in dairy animals if the udder is kept clean and dry [3]. It is important to educate the livestock handlers about the udder hygiene [44].

\section{Conclusion}

Rhodotorulosis caused by several species of Rhodotorula is an emerging opportunistic fungal disease of humans as well as well animals. The disease is reported from developing and developed nations of the world. Infection due to Rhodotorula especially in immunosuppressed individuals can be life threatening if the treatment is delayed. Direct demonstration of fungal agent in the clinical samples and its isolation in pure and luxuriant form is considered as gold standard of diagnosis. Correct and prompt diagnosis and immediate therapy is imperative to mitigate the suffering in immunocompromised patients. Further research work on the pathogenesis, risk factors, molecular epidemiology, diagnosis and therapy is emphasized. As the fungus has been isolated from dairy products, fruits, and peanuts, the role of food as a source of Rhototorula infection needs to be elucidated. Additional studies should be conducted to ascertain the etiologic significance of Rhodotorula species in some more clinical disorders of humans and animals.

\section{Acknowledgements}

The authors are very grateful to Prof. Dr. R.K.Narayan for his suggestions during the preparation of manuscript and Anubha Priyabandhu for computer help.

\section{Contribution of authors}

All the authors contributed equally. They read the final version, and approved it for publication.

\section{Conflict of Interest}

The authors declare that they have no conflict of interest.

\section{References}

1. Hazen KC (1995) New and emerging yeast pathogens. Clin Microbiol Rev 8(4): 462- 478.

2. Pfaller MA, Diekema DJ (2004) Rare and emerging opportunistic fungal pathogens: concern for resistance beyond Candida albicans and Aspergillus fumigatus. J Clin Microbiol 42(10): 4419- 4431.

3. Pal M (2007) Veterinary and Medical Mycology. $1^{\text {st }}$ (Edn.), Indian Council of Agricultural Research, New Delhi, India.

4. Rose HD, Kurup VP (1977) Colonization of hospitalized patients with yeast-like organisms. Sabouraudia 15(3): 251-256.

5. Dave P, Pal M (2015) New medium "APRM" for isolation of medically important fungi from clinical and environmental samples. International J Livestock Res 5(3): 10-18.

6. Lifshitz T, Levy J (2005) Rhodotorula rubra keratitis and melting after repeated penetrating keratoplasty. Eur J Ophthalmol 15(1): 135-137.

7. Kaur R, Wadhwa A, Agarwal S (2007) Rhodotorula mucilaginosa: an unusual cause of oral ulcers in AIDS patients. AIDS 21(8): 1068-1069.

8. Tuon FF, Costa SF (2008) Rhodotorula infection. A systematic review of 128 cases from literature. Rev Iberoam Micol 25(3):135-140.

9. Gomez-Lopez A, Mellado E, Rodriguez-Tudela JL, CuencaEstrella M (2005) Susceptibility profile of 29 clinical isolates of Rhodotorula spp., and literature review. J Antimicrob Chemother 55(3): 312-316.

10. Goyal R, Das S, Arora A, Aggarwal A (2008) Rhodotorula mucilaginosa as a cause of persistent femoral nonunion. J Postgraduate Medicine 54(1): 25-27.

11. Ragheb RR, Abou-El-Makarem M, Ramzy A, Saleh NA (1998) Some studies on the microbiological causes and biochemical changes in mastitic milk with emphasis on fungi and Mycoplasma. Veterinary Medical J 37(74): 2432.

12. Klimaite J, Paskevicius A, Aniulis E (2003) Etiology yeasts and other microorganism of sub-clinical mastitis in cows. Veterinary and Zootechnics 23(45): 5- 9.

13. Sirohi NS, Khar SK (2000) Mycotic Infection in reproductive disorders of bovine. Ind J Dairy Sci 53: 108111. 


\section{Open Access Journal of Mycology \& Mycological Sciences}

14. Fell JW, Boekhout T, Fonseca A, Scorzetti G, StatzellTallman A (2000) Biodiversity and systematics of basidiomycetous yeasts as determined by large-subunit rDNA D1/D2 domain sequence analysis. Int J Syst Evol Microbiol 50(Pt 3): 1351-1371.

15. Lo Re V, Fishman NO, Nachamkin I (2003) Recurrent catheter-related Rhodotorula rubra infection. Clin Microbiology \& Infection 9(8): 897-899.

16. Larone DH (2002) Medically Important Fungi. $2^{\text {nd }}$ (Edn.), American society for microbiology. Washington, DC, USA.

17. Wirth F, Goldani LZ (2012) Epidemiology of Rhodotorula: an emerging pathogen. Interdiscip Perspect Infect Dis 465717.

18. de Zoysa JR, Searle M, Lynn KL, Robson RA (2001) Successful treatment of CAPD peritonitis caused by Rhodotorula mucilaginosa. Perit Dial Int 21(6): 627-628.

19. Diekema DJ, Petroelje B, Messer SA, Hollis RJ, Pfaller MA (2005) Activities of available and investigational antifungal agents against Rhodotorula species. J Clin Microbiol 43(1): 476-478.

20. Shinde RS, Mantur BG, Patil G, Parande MV, Parande AM (2008) Meningitis due to Rhodotorula glutinis in an HIV infected patient. Indian J Med Microbiol 26(4): 375-397.

21. Thakur K, Singh G, Agarwal S, Rani L (2007) Meningitis caused by Rhodotorula rubra in a human immunodeficiency virus infected patient. Ind J Med Microbiol 25(2): 166-168.

22. Baradkar VP, Kumar S (2008) Meningitis caused by Rhodotorula mucilaginosa in human immunodeficiency virus seropositive patient. Ann Indian Acad Neurol 11(4): 245-247.

23. Fung H B, Martyn CA, Shahidi A, Brown ST (2009) Rhodotorula mucilaginosa lymphadenitis in an HIVinfected patient. Int J Infect Dis 13(1): 27-29.

24. Perniola R, Faneschi ML, Manso E, Pizzolante M, Rizzo A, et al. (2006) Rhodotorula mucilaginosa outbreak in neonatal intensive care unit: microbiological features, clinical presentation, and analysis of related variables, European J Clinical Microbiol Infectious Diseases 25(3): 193-196.

25. Tuon FF, Duboc de Almeida GM, Costa SF (2007) Central venous catheter-associated fungemia due to Rhodotorula spp.--a systematic review. Medical Mycol 45(5): 441-447.

26. Hagan ME, Klotz SA, Bartholomew W, Potter L, Nelson
M (1995) A pseudoepidemic of Rhodotorula rubra: a marker for microbial contamination of the bronchoscope. Infect Control Hosp Epidemiol 16(12): 727-728.

27. Fonseca A, Inacio S (2006) Phylloplane yeasts. Biodiversity and Ecophysiology of yeasts. Springer. Berlin, Germany, pp: 263.

28. Garcia-Suarez J, Gomez-Herruz PJ , Cuadros A, Burgaleta C (2011) Epidemiology and outcome of Rhodotorula infection in haematological patients. Mycoses 54(4): 318-324.

29. Louria DB, Greenberg, SM, Molander DW (1960) Fungemia caused by certain nonpathogenic strains of the family Cryptococcaceae. N Engl J Med 263: 12811284.

30. Gogate A, Deodhar L, Gogate S (1987) Hydrosalpinx due to Rodotorula glutinis (a case report). J Postgrad Med 33(1): 34 .

31. Aruo SK (1980) Necrotizing cutaneous rhodotorulosis in chickens in Uganda. Avian Diseases 24(4): 1038-1043.

32. Monga DP, Garg DN (1980) Ovine pulmonary infection caused by Rhodotorula rubra. Mykosen 23(4): 208-211.

33. Kadota K, Uchida K, Nagatomo T, Goto Y, Shinjo T, et al. (1995) Granulomatous epididymitis related to Rhodotorula glutinis infection in a dog. Vet Pathol 32(6): 716-718.

34. Alvarez-Perez S, Mateos A, Dominguez L, MartinezNevado E, Blanco JL, et al. (2010) Isolation of Rhodotorula mucilaginosa from skin lesions in a Southern sea lion (Otaria flavescens): a case report. Veterinarni Medicina 55: 297-301.

35. Costa EO, Gandra CR, Pires MF, Coutinho SD, Castilho W, et al. (1993) Survey of bovine mycotic mastitis in dairy herds in the State of São Paulo, Brazil. Mycopathologia 124(1): 13-17.

36. Duarte ER, Resende JCP, Rosa CA, Hamdan S (2001) Prevalence of yeasts and mycelial fungi in bovine parasitic otitis in the state of Minas Gerais, Brazil. J Vet Med B Infect Dis Vet Public Health 48(8): 631-635.

37. Costa AF, Sidrim JC, Cordeiro RA, Brilhante RSN, Monteiro AJ, et al. (2010) Urban pigeons (Columba livia) as a potential source of pathogenic yeasts: a focus on antifungal susceptibility of Cryptococcus strains in Northeast Brazil. Mycopathologia 169(3) 207-213.

38. Pal M (1997) Use of Pal's sunflower seed medium for an early diagnosis of cryptococcosis. The Antispetic 95(5): 
175.

39. Pal M, Hasegawa A, Ono K, Lee CW (1990) A new staining solution for the morphological studies of fungi and Prototheca. Japanese J Vet Res 52(3): 527-531.

40. Pal M (2004) Efficacy of Narayan stain for the morphological studies of moulds, yeasts and algae. Rev Iberoam Micol 21(4): 219.

41. Lunardi LW, Aquino VR, Zimerman RA, Goldani LZ (2006) Epidemiology and outcome of Rhodotorula fungemia in a tertiary care hospital. Clin Infect Dis 43(6): e60-e63.

42. Zaas AK, Boyce M, Schell W, Lodge BA, Miller JL, et al. (2003) Risk of fungemia due to Rhodotorula and antiungal susceptibility testing of Rhodutorula isolates. J Clin Microbiol 41(11): 5233-5235.

43. Khodavaisy S, Nabili M, Davari B, Vahedi M (2011) Evaluation of bacterial and fungal contamination in the health care workers' hands and rings in the intensive care unit. J Prev Med Hyg 52(4): 215-218.

44. Pal M, Kerorsa GB, Marami LM, Kandi V (2007) Epidemiology, Pathogenicity, Animal Infections, Antibiotic Resistance, Public Health Significance, and Economic Impact of Staphylococcus Aureus: A Comprehensive Review. American Journal of Public Health Research 8(1): 14-21. 